\title{
Le corps éclaté : Antoine et Cléopâtre, Coriolan, et Othello
}

\section{Caroline Di Miceli}

\section{(2) OpenEdition}

\section{Journals}

\section{Édition électronique}

URL : http://journals.openedition.org/shakespeare/1186

DOI : 10.4000/shakespeare.1186

ISSN : 2271-6424

\section{Éditeur}

Société Française Shakespeare

\section{Édition imprimée}

Date de publication : 1 novembre 1991

Pagination : 17-28

\section{Référence électronique}

Caroline Di Miceli, «Le corps éclaté : Antoine et Cléopâtre, Coriolan, et Othello 》, Actes des congrès de la Société française Shakespeare [En ligne], 9 | 1991, mis en ligne le 01 janvier 2007, consulté le 06 mai 2019. URL : http://journals.openedition.org/shakespeare/1186; DOI : 10.4000/shakespeare.1186 


\section{SHAKESPEARE \\ ET LE CORPS A LA RENAISSANCE}

Société Française Shakespeare

Actes du Congrès 1990

sous la direction

de

M.T. JONES-DAVIES

PARIS

LES BELLES LETTRES

1991 
Tous droits de traduction, de reproduction et d'adaptation réservés pour tous les pays.

(C) 1991 Société d'édition Les Belles Lettres, 95 bd Raspail 75006 Paris

ISBN $2.25169117-\mathrm{X}$ 


\section{Le corps éclaté Antoine et Cléopâtre, Coriolan et Othello}

The breaking of so great a thing should make

A greater crack.

(V. i. 14-15)

Ainsi s'étonne Octave à l'annonce de la mort d'Antoine; mort d'un homme de stature héroïque, guerrier redoutable, déchiré entre la sensualité de l'Égypte et la raison d'État de Rome. L'image du corps éclaté est essentielle dans la tragédie. Elle exprime à la fois le désordre du macrocosme et du microcosme. Elle est le reflet concret du pouvoir destructeur des forces tragiques. Octave voit dans la mort d'Antoine un événement à l'échelle du globe. L'éclatement du corps aurait du s'accompagner d'un cataclysme de grandeur comparable, un tremblement de terre qui aurait affecté l'ordre du monde.

Comme Antoine et Cléopâtre, Coriolan, l'autre pièce romaine, s'appuie sur cette image du corps éclaté. Si, dans la première tragédie, les images de morcellement gravitent surtout autour du corps d'Antoine, dans la seconde, par contre, elles concernent essentiellement le corps d'État.

Dès le début d'Antoine et Cléopâtre, nous voyons que le corps puissant du héros a été détourné de sa fonction première le combat - par le pouvoir abusif de la beauté sensuelle de la Reine. Certaines parties du corps héroïque se sont éloignées et détournées du service de l'État dans la guerre. Elles sont devenues disjointes :

Those his goodly eyes,

That o'er the files and musters of the war, Have glow'd like plated Mars, now bend, now turn

The office and devotion of their view

Upon a tawny front. 
Le pouvoir subversif de lamour entraine le "divorce" entre les parties du corps et leur fonction, définie par Rome. Ayant perdu le but qui les unifiait, elles amorcent une fragmentation d'autant plus redoutable aux yeux des Romains qu'Antoine est assimilé à Atlas, qui portait le poids du monde sur ses épaules : "the demi-Atlas of this earth" (I. v. 23). Il est aussi l'un des membres du triumvirat, "the triple pillar of the world" (I. i. 12), les trois colonnes qui soutiennent l'édifice de l'Empire. Le thème de l'architecture qui parcourt la pièce se trouve donc relié au thème du corps entier ou disloqué.

Antoine est, lui, bien loin de cette impression de morcellement tragique. Le corps amoureux s'oppose à l'unité du corps politique mais devient le reflet de l'unité et de l'harmonie de l'univers. Mieux même, les amants ne voient dans l'univers que le pâle reflet de leur amour :

Eternity was in our lips, and eyes,

Bliss in our brows' bent; none our parts so poor,

But was a race of heaven.

(I. iii. $35-37$ )

Dans la courbe des sourcils apparaît la voûte des cieux, image harmonieuse. Le pouvoir de l'amour confère l'immortalité et l'unité au corps. L'ensemble ne connait plus de limites sauf à les trouver aù-delà de l'univers connu. Cléopâtre dit :

I'll set a bourne how far to be belov'd.

Antoine répond :

Then must thou needs find out new heaven, new earth.

(I. i. 17)

L'amour repousse à l'infini les limites du corps, de la vie et de la mort. Ainsi peu importe à Antoine que l'Empire même s'écroule, il a les bras de Cléopâtre pour espace vital, agrandi par l'amour aux dimensions du macrocosme. Ils englobent l'empire, le monde et l'univers :

and the wide arch

Of the ranged empire fall ! Here is my space.

Vision grandiose, certes, mais image qui ne laisse aucun doute sur l'existence des forces destructrices qui pourraient provoquer l'effondrement de l'arche porteuse de l'empire. Image prémonitoire aussi dans la mesure où, plus tard, Antoine finira par 
souhaiter l'effondrement d'une autre architecture, celle de son propre corps. La gloire des armes, puis l'amour de la Reine liaient ensemble les éléments de l'édifice. Avec leur disparition, le morcellement est inévitable. En effet, lorsqu'il se croit trahi, Antoine sent que son corps perd son unité et même sa forme. Il se compare aux nuages qui flottent dans le ciel sans direction, qui prennent une forme pour se dissiper lentement avant d'en prendre une autre. Ainsi il exprime aussi lidée que son corps partira rejoindre le néant de la mort :

That which is now a horse, even with a thought

The rack dislimns, and makes it indistinct

As water is in water ...

My good knave Eros, now thy captain is

Even such a body : here I am Antony,

Yet cannot hold this visible shape, my knave.

(IV. xiv. 9-14)

A l'annonce de la mort de Cléopâtre, n'ayant plus de raison de maintenir ensemble les différentes parties de son corps, il appelle de ses vœux la rupture de son unité physique :

$O$, cleave, my sides,

Heart, once be stronger than thy continent,

Crack thy frail case.

(IV. xiv. 39-41)

Le cœur, gonflé de chagrin, devrait être plus fort que le reste du corps, en entraîner la destruction totale. Ce coffre frêle qu'est le corps devrait céder devant l'ampleur de la souffrance physique et morale. Dès le vers suivant, la rupture est consommée :

bruised pieces, go

You have been nobly borne.

(IV. xiv. 42-43)

"Pieces", le corps est brisé.

Cléopâtre utilise une image de même nature en évoquant sa mort. Son corps est une maison, lien avec le thème de l'architecture, qu'elle démolira elle-même, "This mortal house I'll ruin". Du corps morcelé, ses esprits légers pourront s'échapper pour prendre leur envolée :

I am fire, and air; my other elements

I give to baser life.

(V. ii. 288-289) 
Elle tente de recréer la stature héroique d'Antoine par la vision de son corps qu'elle décrit à Dolabella :

His face was as the heavens, and therein stuck

A sun and moon, which kept her course, and lighted

The little $O$, the earth.

(V. ii. 79-81)

Antoine est le soleil et la lune du macrocosme, qui illuminent le monde, sans jamais le laisser plongé dans l'obscurité. Il est ici la personnification de l'harmonie céleste, l'équilibre parfait entre nuit et jour. C'est son corps qui règle le cours des planètes, qui donne son rythme à l'univers. Antoine est le colosse, la merveille du monde, qui enjambe océans et continents. La courbe de son bras levé est comme la voûte des cieux :

His legs bestrid the ocean, his rear'd arm

Crested the world : his voice was propertied

As all the tuned spheres.

(V. ii. 82-84)

Le corps d'Antoine devient ainsi la clé de voûte de l'arche du monde et de l'univers. Cléopâtre reprend Dollabella, qui essaie de la ramener à la réalité en niant l'existence d'un tel homme. Il existe, puisque créé par son imagination, et, ce corps, produit de la vitalité de l'imagination passionnée a plus de réalité qu'un vrai corps :

But if there be, or ever were one such,

It's past the size of dreaming : nature wants stuff

To vie strange forms with fancy, yet to imagine

An Antony were nature's piece, gainst fancy

Condemning shadows quite.

(V. ii. $96-100)$

Ce corps imaginé dépasse la taille même des rêves, il n'y a plus de mesure possible. Au-delà des rêves, au delà de l'univers, le corps d'Antoine, fragmenté par les forces tragiques, a été recréé dans le royaume de l'imaginaire. Cette reconstruction du corps morcelé est le rempart érigé par Cléopâtre contre la destruction du corps par la mort, et le vide qu'elle entraîne. Les deux corps hérö̈ques, separés un instant sur terre, se rejoindront lorsque Octave décidera, dernier hommage à leur stature de géants, de les réunir dans le même tombeau. 
L'autre pièce romaine, que je vais traiter, Coriolan, utilise aussi l'image du corps éclaté, mais elle est essentiellement reliée à celle du corps politique et la notion de reconstruction apparaît de façon moins nette. C'est la fable du ventre qui est centrale : autour d'elle gravitent toutes les images corporelles. Le corps unifié représente un idéal politique, l'intercommunication et l'interdépendance souhaitables des classes sociales. A l'époque, les différentes parties du corps étaient classées en deux catégories : les parties "nobles" qui comprenaient le cœur, les poumons et le foie, puis les parties "viles", qui comprenaient les organes de reproduction et de digestion. Dans la fable de Menenius, néanmoins, c'est l'estomac qui occupe la place centrale, puisque par lui passe la nourriture destinée à chaque partie du corps. Situé au milieu du corps, le ventre constitue un lieu stratégique autour duquel gravitent les "membres", les plébéiens qui travaillent loin du centre de pouvoir. Le corps humain est comparable à une forteresse dont l'estomac est le sénéchal, occupé à distribuer les vivres à chaque membre de la communauté, selon son rang, et dans les différentes pièces du château-fort :

And through the cranks and offices of man,

The strongest nerve and small inferior veins

From me receive that natural competency

Whereby they live.

En fait, l'idéal du corps politique unifié est mis en cause par la révolte des plébéiens et Menenius se sert de cette fable pour essayer de les convaincre qu'elle est vouée à l'échec, puisqu'elle va contre l'ordre naturel. Je citerai la partie centrale de la fable :

There was a time when all the body's members

Rebelled against the belly, thus accused it :

That only like a gulf it did remain

I' th' midst $o^{\prime}$ th' body, idle and inactive,

Still cupboarding the viand, never bearing

Like labour with the rest, where th'other instruments

Did see and hear, devise, instruct, walk and feel,

And, mutually participate, did minister

Unto the appetite and affection common

Of the whole body.

Représentant les nobles de la ville, Menenius met l'accent sur l'unité nécessaire de toutes les parties du corps, qui par leur travail 
commun le maintiennent "entier", "the whole body". Grâce à sa fable et aussi à un échange de plaisanteries avec les plébéiens, il parvient à rétablir momentanément l'unité, et à faire accepter la position privilégiée de l'estomac: "i'th'midst o'th'body". Cependant, l'estomac, accusé à tort de profiter de sa situation, répond avec mépris aux accusations des membres "inférieurs" :

it tauntingly replied

To the discontented members, the mutinous parts

L'utilisation du mot "parts" introduit la notion de division que la fable voulait combattre. Dans la pièce, on souligne toujours la place inférieure des plébéiens, éloignés en distance et en importance, separés du corps central. Ce sont des mains, des bras, des langues, des bouches, juste bons à travailler. Ce sont des parties isolées, disjointes, n'ayant aucun lien avec le tronc du corps politique. De façon ironique, Menenius appelle le premier citoyen "grand orteil" de l'assemblée, "the great toe" (I. i. 153), lui attribuant ainsi la place la moins importante et certainement la plus éloignée du centre de pouvoir.

Le morcellement du corps politique est accentué par l'arrivée de Coriolan qui entre en scène avec fracas. Les injures qu'il lance aux plébéiens élargissent encore le fossé qui se creuse entre eux et les patriciens. Son langage, d'où la rhétorique qui caractérisait le style de Menenius est absente, ne vise ni à communiquer, ni à convaincre. Il renvoie les citoyens chez eux en ces termes significatifs: "Go, get you home, you fragments." (I. i. 220). Le corps éclate. Il n'est pas surprenant, dès lors, que plus tard Coriolan, lui-même "membre" du corps politique soit rejeté, étant devenu aux yeux de la plèbe gangreneux, inutile et dangereux pour la santé de l'État. Les deux tribuns conseillereont l'amputation immédiate :

The service of the foot,

Being once gangrened, is not then respected

For what it before was.

(III. i. 304-306)

Ce qui est intéressant c'est que la comparaison montre clairement le changement de statut de Coriolan. Il est devenu "pied", aussi éloigné du cœur battant du corps politique que le grand orteil, et aussi inutile.

Le corps du héros est un des pivots de la pièce. Par sa présence dans la ville, il soutient le corps politique, à cause de son 
absence, quand il en est rejete, le corps politique risque de se désintégrer. D'abord, pour servir l'État, Coriolan a transformé son corps en formidable machine du guerre, sans pitié, inexorable dans son avancée à travers les lignes ennemies. Ce corps, si endurci, si impénétrable, est le bélier qui forcera les portes des villes. C'est une épée : Coriolan crie à ses soldats, "make you a sword of me" (1. vi. 76). Son corps perd de son humanité, ne relève plus de l'ordre naturel, il devient une chose, à croire le langage des autres lorsqu'ils doivent parler de lui, et, ne trouvant aucun terme adéquat, se rabattent sur "thing", "he was a thing of blood" (II. ii. 107). Ses ennemis admirent son invincibilité. Comme l'indique Aufidius, son corps reste intact, même devant l'assaut des armes. Ce n'est pas le corps qui redoute l'épée mais le métal de l'épée qui se forge contre le corps-enclume: "Here I clip the anvil of my sword" (IV. v. 112-113). Ce n'est pas le corps qui est brisé, mais la lance :
Let me twine
Mine arms about that body, where against My grainèd ash an hundred times hath broke And scarred the moon with splinters.

(IV. v. 109-112)

Cependant, le corps peut être entaillé. Dans les cicatrices, rituellement exposées aux yeux de la plèbe lors de l'élection des consuls, nous voyons les germes d'un éclatement possible. Sa mère dit: "There will be large cicatrices to show the people ... He received in the repulse of Tarquin seven hurts in the body" et Menenius reprend "one i'th'neck and two i'th'thigh - there's nine that I know." (II. i. 141-146).

Si le corps de Coriolan soutient le corps politique et contribue à son unité dans la guerre, son langage, par contre, contribue à sa fragmentation. De l'aveu de Coriolan, il n'a aucun don pour le discours, il craint la rhétorique puisqu'elle risque de détourner son corps de sa fonction première - le combat :

My throat of war be turned

Which choired with my drum, into a pipe

Small as an eunuch or the virgin voice

That babies lulls asleep! The smiles of knaves

Tent in my cheeks and the schoolboy's tears take up

The glasses of my sight! A beggar"s tongue

Make motion through my lips, and my armèd knees,

Which bowed but in my stimup, bend like his 
That hath receives alms!

(III. ii. $112-120$ )

Cependant l'art de la rhétorique est liê à l'art de gouverner et sert aussi le corps politique, comme l'a démontré Menenius, qui l'a utilisé pour maîtriser la foule. C'est une force d'ordre. Or Coriolan ne peut parler sans injurier : il crie aux plébéiens, après leur refus de l'élire:

You common cry of curs, whose breath I hate

As reek o'th'rotten fens.

(III. iii. 120-121)

You herd of . . - Boils and plagues

Plaster you o'er ...

... Pluto and hell!

(I. iv. 31-36)

Nous verrons aussi dans Othello que cette fragmentation du discours, cette absence de liens logiques entre les phrases sont les signes précurseurs de l'effondrement du corps même. Les thèmes de la nourriture et de la digestion évoqués par Menenius continuent dans la pièce. L'action dramatique se situe par rapport aux murs de la ville. Il y a beaucoup d'entrées et de sorties. Coriolan prend de force la ville de Corioles. Plus tard il est expulsé de sa propre ville, conduit loin des murs, image de l'expulsion du ventre de la mère, puisque c'est à ce moment qu'il décide de rompre avec Rome, et ainsi avec sa mère et sa famille. Il entre secrètement dans la ville d'Aufidius, à la recherche d'un autre corps à servir, et de là aussi il sera rejeté brutalement par la mort. Ceci est aussi le thème de l'évacuation, suite logique de la digestion.

Sans le soutien du corps de Coriolan, invincible, la structure du corps politique romain risque de se disloquer complètement, avec la destruction totale de la ville, mise à feu et à sang. Ce corps redoutable soutient un moment le camp d'Aufidius, et puis, réintègre le corps politique premier, retrouve un instant avant de mourir les liens primitifs qui le lient à sa mère et à sa ville. Rome ne peut être sauvée que par le sacrifice d'un de ses fils. Volumnia, qui avait toujours renié les liens naturels entre mère et fils (disant, par exemple, que le sang qui jaillissait du front d'Hector au combat était plus beau à voir que le lait qui nourrissait son fils), est forcée à la fin de reconnaître ces liens corporels, se proclamant terre-mère, piétinée par les armées de son fils. De façon étonnante, c'est l'indication scénique qui clôture leur entretien qui représente la tentative de reconstruction du corps. "Holds her by the hand, silent" ( $V$. iii). Coriolan prend sa mère par la main. 
Dans ce geste sont réaffirmés les liens corporels. Le cordon ombilical qui fut coupé quand Coriolan avait été expulsé de Rome, est renoué, et l'espace d'un instant, le corps du fils est relié à la fois à celui de la mère et à celui de l'État.

Dans Othello, l'image centrale de la tragédie est celle du corps sacrifié de la femme dont la beauté et la pureté même deviennent dans l'esprit d'un mari jaloux, autant de masques diaboliques de la plus profonde corruption intérieure.

Othello se déroule dans un lieu quasiment clos : la lagune de Venise, ses ruelles la nuit, lîle de Chypre, et surtout la chambre nuptiale. Traditionnellement située dans l'alcôve de la scène élisabéthaine, c'est par excellence l'espace domestique à l'écart de la scène principale. C'est un aspect qui est fortement souligné par la mise en scène de la $\mathrm{BBC}$ où l'on voit toujours les portes se refermer derrière le couple. Les regards indiscrets des spectateursvoyeurs violeraient l'intimité conjugale. Dans son article "Let It be Hid", Lynda Boose insiste sur le rapprochement que l'on peut faire entre l'espace clos de la scène et l'espace clos du corps. Je cite :

From the opening scene of this play, to be a spectator is to be invited into an Iago-orchestrated world of images centered upon the availability of Desdemona's body - the locus where, inexorably, the action of the drama likewise leads. ${ }^{1}$

Le corps de Desdémone, vierge, était nécessairement intact; après le mariage, la brèche ayant été ouverte, son intégrité se reconstruit par la fidélité. Le mouchoir, si célèbre, si discuté, est un prolongement de limage du corps. C'est une extension des draps nuptiaux après la nuit de noces, blancs, tachés de rouge "spotted with strawberries". Ce mouchoir devient le symbole du corps de la femme, pur, entier, possédé par un seul homme. Dans son innocence, Desdémone parle de son corps comme d'un vase précieux "vessel" dont elle réserve l'usage à son mari, "if to preserve this vessel for my lord" (IV. ii. 85). Récipient des bienfaits d'un époux légitime auquel elle se sait fidèle, il est intact et sans faille. Desdémone demande à Emilia de faire son lit avec ses draps de noces pour qu'ils soient son linceul si elle venait à mourir. Enveloppé dans ces draps qui symbolisent l'unité et l'union perdues, son corps retrouvera après sa mort son intégrité première. Othello est, par excellence, la tragédie du morcellement du corps, dominé par une des ses parties. Iago, la langue qui abuse et Othello, l'oreille qui 
écoute feront de Desdémone une main qui touche, une paume moite :

Hot, hot, and moist, this hand of yours requires

A sequester from liberty.

(III. iv. 35-36)

L'oreille trop ouverte d'Othello, trop tendue dans la mauvaise direction, est sa faille tragique, l'hamartia, la cause de la tragédie. Iago dit :

I'll pour this pestilence into his ear,

That she repeals him for her body's lust.

(II. iii. 347-348)

Echo de Hamlet. Comme l'écrit Marie-Christine Pouchelle :

Dans le corps-forteresse, tout orifice était une faille par laquelle le monde extérieur menaçait sans cesse l'intégrité de la personne, et par laquelle, en sens inverse, risquait de s'échapper l'esprit vital, voire l'âme même. 2

Une fois amorcée, la désintégration fait des ravages dans plusieurs directions. Dans l'esprit et le corps d'Othello lui-même un morcellement a lieu. Dans son esprit, torturé par la jalousie, toutes les indications et les fausses preuves données par lago s'enchevêtrent. Il est dérouté par des images contradictoires du corps de Desdémone : corps pur et lascif à la fois. La continuité de sa pensée est brisée par un déluge d'images obsessionnelles qui se succèdent rapidement. Comme dans Coriolan, la fragmentation affecte le discours, qui devient hâché, délirant. La déraison est peceptible dans l'absence de tout lien logique dans la phrase. Mais ici Othello perd aussi le contrôle de son corps. Il s'écroule, terrassé par une crise d'épilepsie. Le lien entre corps et esprit brisé, c'est l'effondrement du corps. La perte du sens entraîne la perte des sens, mais auparavant c'est le corps de Desdémone qui a été morcelé :

Pish! Noses, ears and lips. Is't possible ? - . -

Confess ? - - Handkerchief ? - - - O devil !

(IV. i. $42-43$ )

De façon frappante, les parties du corps ainsi disjointes évoquent nécessairement le mouchoir, symbole contradictoire de l'intégrité et de la trahison. Et la vengeance ne se conçoit qu'en termes de dépeçage :

I'll tear her all to pieces.

(III. iii. 438)

et

I'll chop her into messes.

(IV. i. 196) 
Image cannibalesque, réduisant le corps à des plats de viande.

Assez paradoxalement, alors qu'Othello est convaincu de l'infidélité de Desdémone, il ne peut complètement écarter des images relatives à la perfection de sa femme et à sa pureté inhérente. Pour finir, il se résout à ne pas détruire cette perfection :

Not scar that whiter skin of hers than snow, yet Ill not shed her blood,

And smooth as monumental alabaster;

Dans l'image arrive d'abord la notion de blancheur, associée à l'innocence. Le sang et la neige rappellent les couleurs symboliques du mouchoir, blanc et rouge. Mais par l'intermédiaire du marbre blanc l'innocence est reliée au froid de la mort. Même avant d'avoir compris son erreur, son esprit reconstruit le corps que l'esprit jaloux avait morcelé. Deux images sont importantes : celle de la "chrysolite" et celle de la perle. Intuitivement son langage exprime la vérité qu'il ne veut pas encore admettre:

had she been true

If heaven would make me such another world

Of one entire and perfect chrysolite,

I'ld not have sold her for it.

(V. ii. 144-148)

Avec la prétendue infidélité de sa femme, le corps et le monde avaient volé en éclats. Maintenant, dans son esprit la reconstruction a lieu, mais appartient à un autre monde hypothétique, inaccessible; le corps de sa femme est le centre de cet univers fictif. Sûr de la vérité, il utilise l'image de l'Indien, qui, comme lui, a jeté une perle plus précieuse que toute sa tribu:

Like the base Indian, threw a pearl away,

Richer than all his tribe :

(V. ii. 347-349)

Depuis l'Antiquité la perle était l'image de la virginité. Par sa rondeur parfaite, dans le poème du Moyen Age, Pearl, elle était le symbole de la Vierge Marie et du royaume des cieux, éternel et infini. Opaque, lisse, blanche et nacrée, comme la peau de la femme, la perle est féminité même. Le morcellement, dû à la jalousie, était l'œuvre diabolique de lago. Par ces images de la perle et de la chrysolite Othello montre son désir de retrouver une image unifiée du corps de sa femme. Bien que Desdémone pardonne à son mari et 
s'accuse elle-même, on ne pourra pas parler de réconciliation, impropre au genre tragique, mais simplement d'apaisement.

Ainsi dans les trois pièces étudiées, nous constatons que le corps éclaté est une image essentielle à la tragédie. A travers elle s'exprime clairement lidée du désordre social et personnel que les forces tragiques entraînent. Mais Shakespeare tente toujours une reconstruction du corps éclaté, ne serait ce qu'au niveau de la poésie ou des métaphores scéniques. C'est un des éléments qui concourt à créer le retour à l'ordre qui caractérise la fin des tragédies.

CAROLINE DI MICELI

\section{NOTES}

Les citations sont tirées des éditions suivantes :

Shakespeare, Antony and Cleopatra, The Arden Edition, edited by M. R. Ridley, Methuen and Co Ltd, London, 1965.

Shakespeare, Coriolanus, The New Penguin Shakespeare, edited by

G. R. Hibbard, Penguin Books Ltd, Hammondsworth, 1967.

Shakespeare, Othello, The Arden Edition, edited by M. R. Ridley, Methuen and Co Ltd, London, 1965.

1. Lynda Boose, "Let it be Hid", dans Autour d'Othello, Presses de l'UFR Clerc Université Picardie, Colloque organisé par le C.E.R.C.A. 1987 , p. 141.

2. Marie-Christine Pouchelle, Corps et Chirurgie à l'Apogée du Moyen Age, Flammarion, Paris, 1983, p. 154. 